\title{
LA PRUEBA DE ACCESO A LA UNIVERSIDAD COMO REGULADORA DEL PROCESO DE ENSEÑANZA/APRENDIZAJE DE HISTORIA
}

\author{
THE UNIVERSITY ACCESS TEST AS A REGULATOR OF THE TEACHING/LEARNING \\ PROCESS OF HISTORY
}

María del Mar Bernabé Villodre ${ }^{1}$

RESUMEN: En España, el alumnado de segundo curso de Bachillerato se encuentra en un momento complicado por las presiones que suponen las Prueba de Acceso a la Universidad (PAU) y la nota de corte para acceder a la especialidad de su agrado. Esta situación lleva a que el profesorado de esta etapa educativa se plantee la necesidad de adecuarse a los parámetros del citado examen de acceso, con la intención de que los resultados del mismo mejoren y el alumnado pueda acceder a la especialidad deseada. Desde esta consideración, en un centro de la provincia de Alicante, se orientó la asignatura de Historia de España a la superación del examen de acceso a la universidad, y se pudo comprobar una mejora muy significativa de las notas respecto a las calificaciones obtenidas anteriormente, con un sistema más tradicionalista que no seguía el modelo de examen establecido para la PAU por la legislación vigente. Esta metodología posibilitó la mejora de las notas individuales y globales del centro, además de que permitió demostrar que es imprescindible establecer los mismos mecanismos de evaluación en Bachillerato que en las Pruebas de Acceso a la Universidad, para dotar de coherencia esa preparación del acceso a la universidad.

Palabras clave: Historia de España. Didáctica de la Historia. Bachillerato. PAU.

\begin{abstract}
In Spain, the students of second year of Bachillerato are in a moment complicated by the pressures that the University Access Test (PAU) and the cut note to access to the specialty of their liking. This situation leads to the teachers of this educational stage to consider the need to adapt to the parameters of the said access examination, with the intention that the results of the same improve and the students can access the desired specialty. From this consideration, in a center of the province of Alicante, the subject of History of Spain was oriented to the passing of the examination of access to the university, and it was possible to verify a very significant improvement of the grades with respect to the qualifications obtained previously, with a more traditional system that did not follow the model of examination established for the PAU by the current legislation. This methodology made it possible to improve the individual and global grades of the center, in addition to showing that it is essential to establish the same evaluation mechanisms in Bachillerato as in the University Access Tests, in order to provide coherence in this preparation of access to the college.
\end{abstract}

Keywords: History of Spain. Teaching History. High School. PAU.

1 Profesora Ayudante Doctora de la Universidad de Valencia. Doctorado en Teoría e Historia de la Educación. 


\section{Introducción}

La etapa de Bachillerato es decisiva no sólo porque sus calificaciones forman parte de la nota de acceso a la universidad, sino porque el profesorado debe contribuir a que el alumnado mejore su capacidad de reflexión, de síntesis, en definitiva, que le ayude a madurar académica y personalmente. En la etapa de Bachillerato, cuando fue realizada esta investigación (a caballo entre la LOGSE y la $L O E$, aplicada en el curso 2006/2007) y en segundo curso, el alumnado cursaba diferentes materias que luego seleccionaba para realizar en las Pruebas de Acceso a la Universidad (PAU, en adelante). En primer curso, se estudiaba Educación Física, Lengua Castellana y Literatura, Filosofía, Lengua Extranjera, Religión/Alternativa, tres materias optativas según modalidad (estas modalidades eran: Artes, Ciencias de la Naturaleza y de la Salud, Humanidades y Ciencias Sociales, y Tecnología) y una optativa (ofertada por cada centro). Ya, en segundo curso, se estudiaba Historia de España, Lengua y Literatura Castellana, Lengua Extranjera, Religión/Alternativa, tres materias optativas según modalidad (estas modalidades eran: Artes, Ciencias de la Naturaleza y de la Salud, Humanidades y Ciencias Sociales, y Tecnología) y una optativa (ofertada por cada centro).

La disyuntiva a la que se enfrenta el profesorado de ese segundo curso pasa por decidir cuál es la metodología más adecuada para, por un lado, explicar sus contenidos y que éstos sean comprendidos por el alumnado; y, por otro lado, que obtengan una buena calificación en la PAU. Esta situación que para el alumnado resulta tan clara, como muestran autores como Suárez (2010), no parece serlo tanto para el profesorado, ya que la rutina metodológica de trabajo siempre resulta complicada de cambiar.

Ciertamente la adaptación del profesorado al modelo de evaluación de la PAU implica que, si se quieren obtener mejores resultados, debe profundizarse en cada una de sus partes, así como encargar muchos comentarios de texto al alumnado para practicar; lo que, desgraciadamente implica centrarse en preparar exclusivamente ese examen, obviándose que eso no es lo que debería hacerse, como defendía Pérez (2014). Como se verá en epígrafes posteriores, 
esto fue justo lo que se hizo con el alumnado, ya que tuvieron que entregar (obligatoriamente) tres comentarios de texto de cada núcleo temático.

La presión para el alumnado era muy fuerte, ya que se jugaban el acceso a la especialidad de su preferencia. Tanto en segundo curso como en la PAU, debían esforzarse por subir sus notas, en caso de que las calificaciones de primer curso no fuesen todo lo buenas que hubiesen querido: la nota media de ambos cursos se suma a la media de los distintos exámenes realizados durante la PAU. $\mathrm{Y}$, precisamente por ello, el alumnado necesitaba ser formado desde y para ese examen, puesto que su acceso a la universidad dependía de los resultados del mismo. Pero, este último curso de estudios secundarios también tenía un objetivo que el profesorado debía cumplir: completar su proceso de madurez, acorde con su edad y con las exigencias de sus posteriores estudios superiores. García, Jiménez y Moreno (2011) señalaban que el modelo de examen de la PAU promueve la reflexión sobre la conciencia histórica de los futuros estudiantes universitarios; además de que potencia las habilidades sociales de los estudiantes (GÓMEZ; MILLARES, 2013).

La PAU está pensada precisamente para que el alumnado demuestre su madurez general (cognitiva y personal) mediante un examen que no sólo permita mostrar sus conocimientos específicos sobre tal o cual materia, sino que se vea su grado de madurez para integrar la información a partir de textos relacionados con la época y que pueda relacionarlos con conocimientos propios. En este sentido, el profesorado de $2^{\circ}$ de Bachillerato debía orientar y preparar al alumnado para que hiciese frente a las exigencias del examen, al tiempo que, a las exigencias de la etapa educativa, claramente relacionadas. Pérez y Guitián (2006) consideraban que había que dotar al alumnado de los recursos para poder acceder al conocimiento de forma analítica, sintética y objetiva; todo esto se conseguiría si se partiese de la propuesta de examen de PAU, tal como se verá seguidamente.

En los siguientes epígrafes se mostrará cómo la didáctica de la historia no tenía porqué estar reñida con las necesidades del alumnado, en clarísima relación con las exigencias de la PAU. Así como también implicará una adaptación a las capacidades del alumnado (INAREJOS, 2013), puesto que los materiales y la metodología (LLÁCER, 2007) debían ser adecuados para éstos. 


\section{Estado de la cuestión}

El profesorado de Bachillerato sea cual sea su especialidad, se enfrenta con un gran dilema: ¿debe trabajar su materia en una línea tradicional o debe ceñirse a las indicaciones de la PAU? Es decir, si trabaja en sus clases con el mismo planteamiento que en el resto de cursos o si, por el contrario, trabaja la materia desde el planteamiento de evaluación que se indica para la PAU. García, Jiménez y Moreno (2011) consideran que no se puede decir que el profesorado de Bachillerato cambie/adecúe su forma de trabajo por el hecho de tener que realizar el modelo concreto propuesto en la PAU, en la misma línea de lo que defiende Pérez (2014) cuando considera que el profesorado parece limitarse a preparar para ese examen, en lugar de enseñar Historia de España.

Como se verá en epígrafes posteriores, desde nuestra experiencia en este nivel y en la asignatura de Historia de España, la respuesta fue clara: había que trabajar a partir del examen de la PAU para que el alumnado estuviese realmente preparado para superar esas pruebas y poder cursar los estudios de su preferencia. Aunque, al tiempo, no se olvidó que se debía favorecía su madurez y capacidad de reflexión gracias al trabajo de textos relacionados con los núcleos temáticos.

\subsection{La didáctica de la Historia}

La enseñanza en la educación obligatoria, en cualquiera de sus etapas, es totalmente diferente de la realizada en la etapa postobligatoria; no obstante, como señalan Pérez y Guitián (2006) o Acosta (2010), el modelo didáctico principal para una y otra etapa no deja de ser transmisivo en su mayoría.

A pesar de toda esa fuerte tradición o tendencia comentada, didácticamente hablando se debía partir del hecho de que, a nivel evolutivo, el alumnado se encontraba en un momento en el que se debía potenciar su madurez y su reflexión. Como señalaban García y Lara (2009), en esta etapa había que tener en cuenta su capacidad de abstracción e inferencia del 
pensamiento formal. Debido a esto, el profesorado tenía que enfocar el proceso didáctico de modo que el alumnado desarrollase estas capacidades, que le permitirían acceder a los estudios superiores y desarrollarlos con mayor soltura.

Para desarrollar este tipo de proceso de enseñanza/aprendizaje acorde con las necesidades del segundo curso de Bachillerato y de la vecina PAU, no bastaba con que se utilizasen apuntes adecuados o materiales de última tecnología (ORTIZ; MILLARES, 2012), sino que el proceso de llegar a interiorizar unos conceptos históricos clave sería lo determinante para garantizar la comprensión de los mismos por parte del alumnado. Este tipo de ideas eran las que fundamentaban la investigación desarrollada en epígrafes siguientes, que muestra cómo ese planteamiento metodológico y no la entrega de un temario en sí mismo, fue lo que hizo que las calificaciones de la PAU mejorasen.

\subsection{Segundo curso de Bachillerato: Historia de España}

Durante la etapa postobligatoria de Bachillerato, de acuerdo con el Real Decreto 1467/2007, de 2 de noviembre, por el que se establece la estructura del bachillerato y se fijan sus enseñanzas mínimas, se considera que ésta tiene como finalidad proporcionar madurez intelectual y humana, además de social (ESPANHA, 2007). A adquirir la citada madurez intelectual debía contribuir la formación histórica, puesto que el desarrollo de la ciudadanía democrática (objetivo de Bachillerato) sólo podría conseguirse adecuadamente a través de asignaturas como Historia de España.

La asignatura de Historia de España es obligatoria para todo el alumnado, tanto si cursan la rama de conocimiento de Artes y Humanidades, de Ciencias Sociales y Jurídicas, como de Ciencias, de Ciencias de la Salud, o de Ingeniería y arquitectura. Lo que permite justificar ese objetivo de etapa, así como el conocimiento y la valoración crítica de la realidad contemporánea española y europea, otro de los objetivos que son especialmente "adquiribles" desde la asignatura citada.

En la Comunidad Valenciana, el Decreto 102/2008, de 11 de julio, del Consell, por el que se establece el currículo del bachillerato en la Comunitat 
Valenciana, marcaba las líneas de actuación de la asignatura de Historia de España (VALENCIA, 2008). Se consideró que el estudio de esta asignatura permitía profundizar e indagar en la situación actual, desde el estudio que posibilitaba el conocimiento del pasado como explicación del presente. Por supuesto, todo esto justificaba la concreción de un modelo de examen, en el que se intentaba que el alumnado reflexionase, incorporase y relacionase información con madurez.

Los núcleos temáticos comprendidos en la asignatura durante los cursos investigados, fueron los siguientes:

- Las Raíces Históricas de la España Contemporánea.

- La construcción del Estado Liberal.

- La España de la Restauración.

- Dictadura, República y Guerra.

- España durante el franquismo.

- La recuperación democrática. (VALENCIA, 2008).

Desde este temario se pretendía conseguir que el alumnado tuviese capacidad crítica para enfrentarse a su realidad sociocultural. De hecho, uno de los objetivos de esta asignatura, establecido en la anteriormente citada referencia legislativa, se centraba en el fomento de la visión integradora de la historia del país para promover la tolerancia y la solidaridad. Pero esto sólo se podría conseguir si ese estudio de la Historia de España promoviese la reflexión y, por tanto, el trabajo de textos y documentos históricos; ya que el alumnado debía aprender a analizar y valorar las fuentes como promotoras de su capacidad de percepción crítica (ACOSTA, 2010). Y justo este modelo de "trabajo" era el que parecía imponer la PAU, tal como veremos seguidamente.

\subsection{La Prueba de Acceso a la Universidad (PAU)}

Durante la presente investigación estuvo vigente la Ley Orgánica 2/2006, de 3 de mayo, de Educación ( $L O E$ ), que actualmente ha sido sustituida por la Ley Orgánica 8/2013, de 9 de diciembre, para la mejora de la calidad educativa 
(LOMCE). La PAU es realizada cada año en doble convocatoria (junio como primera y julio como segunda); sin embargo, a partir de las normativas estatales generales como la LOGSE (1990), la LOE (2006) o la LOMCE (2013), cada comunidad autónoma desarrolla sus propias normativas de regulación de las mismas, así como de establecimiento de los contenidos a desarrollar durante el Bachillerato. Alarcón (2010) ha realizado un estudio descriptivo comparado entre los distintos modelos de examen de Historia de España de cada comunidad autónoma española, mostrando las importantes diferencias entre unas y otras; aunque, todas parten de una supuesta defensa del proceso de reflexión sobre los contenidos por parte del alumnado.

La $L O E$, en su Artículo 38 centrado en la Prueba de acceso a la universidad, comentaba que el alumnado debía mostrar su madurez académica y cognitiva, lo que sólo podrá conseguirse si se trabajaba desde una metodología muy activa que implicase el trabajo específico del comentario de texto, justo aquello que se exigía en el citado examen. En este sentido, cuando se mencionaba esa madurez cognitiva y académica del alumnado, estaba en clara relación con lo que se espera de un individuo encuadrado en el final de su adolescencia (comprendida por la Organización Mundial de la Salud hasta los 19 años) (ORGANIZACIÓN MUNDIAL DE LA SALUD, 1999), con 18 años: buscan su identidad (HAZEN; SCHOLZMAN; BERESIN, 2008), descubren y afianzan su identidad vocacional (GAETE, 2015) y, sobre todo, desarrollan nuevas habilidades cognitivas (HAZEN; SCHOLZMAN; BERESIN, 2008). Será capaz de racionalizar de forma abstracta, de imaginar sin experimentar, de alcanzar el pensamiento operatorio formal gradualmente $y$, especialmente importante para el examen de PAU, aumentará su capacidad de resolver problemas planteados (GAETE, 2015). En la vigente LOMCE, los objetivos de PAU no han cambiado tanto, puesto que se sigue valorando la madurez académica del alumnado, aunque sí se ha cambiado el enfoque del ejercicio que el alumnado debe realizar, así como su denominación (EBAU, Evaluación de Bachillerato para el Acceso a la Universidad) en algunas Comunidades Autonómicas de España, pero no así en Alicante (Comunidad Valenciana) donde se sigue usando el término PAU.

La PAU del período comprendido entre el año 2000 y 2007 estuvo regulada por las siguientes normativas estatales: el Real Decreto 704/1999, de 30 de 
abril, por el cual se regulan los procedimientos de selección para el ingreso en los centros universitarios de los estudiantes que reúnen los requisitos necesarios para el acceso a la universidad, el Real Decreto 1640/1999, de 22 de octubre, por el cual se regula la prueba de acceso a estudios universitarios, el Real Decreto 69/2000, de 21 de enero, por el que se regulan los procedimientos de selección para el ingreso en los centros universitarios de los estudiantes que reúnan los requisitos legales necesarios para el acceso a la universidad, y el Real Decreto 1742/2003, de 19 de diciembre, por el cual se establece la normativa básica para el acceso a los estudios oficiales universitarios de carácter oficial. Y, en Alicante (Comunidad Valenciana) donde fue realizada esta investigación, las anteriores referencias se concretaron en una normativa autonómica propia: el Decreto 20/2000, de 8 de febrero, del Gobierno Valenciano, por el cual se crean los órganos de gestión de los procesos de acceso a los estudios universitarios, el Decreto 107/2001, de 12 de junio, del Gobierno Valenciano, por el cual se modifica el Decreto 20/2000, de 8 de febrero, de creación de los órganos de gestión de los procesos de acceso a los estudios universitarios, la Orden de 21 de mayo de 2001 de la Conselleria de Cultura y Educación, por la que se regulan los procedimientos de admisión a enseñanzas universitarias en la Comunidad Valencia, el Decreto 41/2005, de 25 de febrero, del Consejo de la Generalitat, por el cual se crea la Comisión Gestora de los procesos de acceso y preinscripción universitaria en las universidades públicas del Sistema Universitario Valenciano.

Atendiendo a esta normativa autonómica, estas pruebas de acceso eran gestionadas por la denominada "Comisión Organizadora de la Prueba de Acceso a la Universidad". Ésta estaba constituida por un titular de la Subsecretaría de la Conselleria de Cultura, Educación y Ciencia de la Comunidad Valenciana, que la presidía; un Vicerrector de cada una de las universidades públicas valencianas (Universidad Jaime I de Castellón, Universidad de Valencia, Universidad Politécnica de Valencia, Universidad de Alicante y Universidad Miguel Hernández de Elche), designado por el Rector de cada una de ellas; un titular de la Secretaría General de la Conselleria de Cultura, Educación y Ciencia de la Comunidad Valenciana; un titular de la Dirección General de Personal y Dirección General de Enseñanzas Universitarias y de Investigación de la Conselleria de 
Cultura, Educación y Ciencia de la Comunidad Valenciana; un titular de la Dirección del Servicio Central de la Inspección Educativa; y el coordinador de las Pruebas de Acceso a la Universidad. Esta última figura era designada de entre el cuerpo de funcionarios docentes universitarios, encargándose de organizar dichas pruebas.

A todas estas figuras, se sumaba una Subcomisión Académica, presidida por un Vicerrector de la Comisión Organizadora, que debía fijar los contenidos académicos encargados por dicha comisión. Entre sus miembros, estaban: el coordinador de las pruebas, un profesor del cuerpo de funcionarios universitarios, un inspector de educación por cada provincia (Castellón, Valencia y Alicante, que constituyen la Comunidad Valenciana) designado por el Consejero de Cultura, Educación y Ciencia de la Comunidad Valenciana, y un director de instituto de Educación Secundaria Obligatoria de cada provincia de la Comunidad Valenciana (designado por el Consejero de Cultura, Educación y Ciencia de ésta).

Desde el curso académico 2005/2006 hasta el curso académico 2008/2009, el examen de Historia de España presentaba el siguiente enunciado:

Compón un texto sobre uno de los temas del programa de Historia a partir del análisis de las fuentes de información incluidas en la propuesta de examen. Recuerda que la composición deberá integrar los conocimientos del alumnado sobre el tema correspondiente y la información facilitada. La valoración de la composición es sobre 10.

Se establecía así que el alumnado no podía limitarse a realizar únicamente un desarrollo del correspondiente núcleo temático, sino que debía demostrar su madurez cognitiva mediante las relaciones del mismo con los dos textos que constituían el examen. Se trataba de demostrar su capacidad de resolver el problema planteado de establecer relaciones entre la información aprendida y la presentada en los documentos aportados: de acuerdo con Gaete (2015), en esta etapa de adolescencia tardía el alumnado tiene capacidad suficiente para resolver problemas planteados. Así, el alumnado conseguía mostrar que no sólo había conseguido los objetivos de Bachillerato, sino que era capaz de reconocer textos de la época, comentarlos y relacionarlos con los conocimientos previamente adquiridos, demostrando así su capacidad de pensamiento hipotético-deductivo (GAETE, 2015). 
En las reuniones de preparación y orientación del profesorado de Bachillerato, realizadas en la universidad, se comentaron los criterios de calificación y se pidió que se expresasen las opiniones sobre el modelo de examen y sobre los contenidos. Así, se pudo conocer cómo iban a ser evaluados los distintos "apartados" del examen, puesto que éste no sólo consistía en terminar preparando un documento a partir de los textos propuestos, sino que había que incluir las ideas principales del mismo (y por tanto el núcleo temático correspondiente), además de incluir los textos dados a lo largo de la información estudiada. Pero esta forma de trabajo no era demasiado conocida o no había sido muy practicada por aquel alumnado que no estuviese acostumbrado a la realización de comentarios de obras literarias (el Bachillerato científico no había realizado este tipo de materias); de manera que se debía promover el estudio de y desde este "formato".

El examen de Historia de España de la PAU promovía la reflexión de un documento a partir de unos conocimientos previos y la interrelación entre los mismos. De modo que, el profesorado de $2^{\circ}$ curso de Bachillerato se veía en la "obligación" de preparar a su alumnado para que pudiese hacer frente a este formato de examen, limitándose a la preparación de este examen (PÉREZ, 2014); hecho que implicaba una metodología didáctica especialmente adaptada para favorecer ese tipo de desarrollo reflexivo y madurativo de los contenidos históricos, y acorde con sus adquiridas habilidades cognitivas (HAZEN; SCHOLZMAN; BERESIN, 2008), tal como se podrá ver en el epígrafe siguiente.

Ahora bien, durante el curso 2009/2010, el modelo y las exigencias del examen variaron y se han mantenido constantes hasta el presente curso 2007/2018, a pesar del cambio legislativo acontecido de LOE a LOMCE. El modelo actual se centra en los siguientes puntos, algo alejados de la propuesta planteada en este artículo, pero con algunos nexos comunes: concreción del tipo de fuente de los textos propuestos, identificación de ideas principales de ambos textos situándolas en el contexto histórico del núcleo temático correspondiente, definir dos conceptos propuestos incluidos en los textos, y dos preguntas centradas en los epígrafes del núcleo temático en cuestión, que deben relacionar con los dos textos propuestos. Son claras las diferencias con el modelo de examen vigente hasta el curso 2008/2009, aunque se observan similitudes 
porque se debe "relacionar" la información del núcleo temático con los textos propuestos.

\section{Propuestas didácticas para trabajar la Historia de España en $2^{\circ}$ de Bachillerato}

Como se ha comentado anteriormente, el examen de Historia de España trataba de favorecer la madurez cognitiva y personal del estudiante, su capacidad de reflexión y síntesis. Se pretendía que supiese relacionar los contenidos aprendidos con documentos de la época y con su realidad cotidiana, que comprendiese cómo se había llegado a la situación actual y cómo mejorarla o no repetir errores pasados. El modelo actual, vigente desde el curso 2009/2010, ha concretado los epígrafes del respectivo núcleo temático a relacionar con los textos propuestos, lo que se traduce en una redacción más sintetizada por parte del alumnado. Así que, un planteamiento metodológico para trabajar con ese modelo de examen variaría un poco respecto al que propuesto durante esta investigación.

De acuerdo con esto y con los parámetros del examen y de la evaluación, el trabajo del alumnado se dividió de la siguiente manera: atendiendo a los seis núcleos temáticos que contaban con distintos (y numerosos) epígrafes, el alumnado debía realizar tres comentarios de texto de anteriores exámenes de PAU para practicar, aunque en algunos casos no habían aparecido nunca y se recurrió al libro de la editorial Edebé, que contaba con gran cantidad de documentos para practicar. Entonces, se realizaron:

-Núcleo Temático II, "La construcción del Estado Liberal": tres textos de PAU de años anteriores.

- Núcleo Temático III, "La España de la Restauración": tres textos de PAU.

-Núcleo Temático IV, "Dictadura, República y Guerra": tres textos de PAU para este núcleo temático que suponía tres pequeños temas totalmente distintos.

-Núcleo Temático V, "España durante el franquismo": tres textos de PAU, nuevamente. 
-Núcleo Temático VI, "La recuperación democrática": tres textos de PAU con un tema que hubo que ampliar para actualizarlo con los últimos datos de la vida política española.

Puesto que el esquema del examen suponía el reparto de puntos comentado en el anterior epígrafe, se consideró imprescindible que las clases no se limitasen a la explicación del tema y de los puntos más importantes del mismo. De este modo, se procedió a realizar un trabajo más especializado que partió del resumen de cada núcleo temático conforme se iban estudiando. Pero este trabajo de la información en sí misma, no se desarrolló de una forma tan simple como pueda parecer: en primer lugar, se explicaba la información y se subrayaban las ideas principales del libro de texto tomado como base; en segundo lugar, debían realizar su propio resumen a partir de la información comentada en el aula; en último lugar, debían tratar de "ahorrar" palabras y (por tanto) tiempo mediante la utilización de fórmulas más simples que permitiesen mostrar la misma información, pero con otras palabras.

Así, una vez trabajado el núcleo temático correspondiente mediante el resumen que les permitiese mejorar su capacidad de síntesis, se procedió a la práctica del parafraseo. Esto les permitió ahorrar palabras y, de esta manera, tiempo a la hora de desarrollar su documento escrito. Había que tener en cuenta que sólo disponían de 90 minutos para realizar su examen, y tenían que decir mucho en poco tiempo, a lo que había que añadir la extracción de ideas y las relaciones entre los documentos dados y la información preparada para el examen. Este trabajo se realizó con correcciones sobre el documento entregado, para que pudiesen ver cómo se podía ahorrar tiempo si se recurría a expresiones similares, pero más breves. Ésta fue la parte más pesada (temporalmente) para el profesorado, ya que suponía corregir resúmenes de muchas páginas de cada miembro de $2^{\circ}$ de Bachillerato, que se dividía en dos cursos, con un total de 39 y 32 miembros en cada uno. Sin embargo, si se quería conseguir unos mejores resultados en sus calificaciones de PAU, se les debía forzar a realizar este trabajo, para que la información no disminuyese, sino que se redujese la expresión de la misma para que se dijese lo mismo con menos palabras. Es decir, que el núcleo temático pasase de 12 páginas a 4-5 (dos folios por delante y por detrás) que sí resultaba factible escribirlas en 90 minutos. 
La siguiente parte del examen que se trabajó fue la extracción de ideas principales, que de acuerdo con los criterios de calificación suponían dos puntos sobre diez de la nota final. Para profundizar en esta parte, se partió de los distintos textos propuestos en exámenes anteriores de PAU $\mathrm{y}$, al no tratarse todos los núcleos temáticos citados, se incluyeron textos del libro de la editorial trabajada en el centro. También se hizo necesario trabajarla de una forma específica para que contasen con muchas opciones de realización y que no todos presentasen los mismos "esquemas", es decir, se indicaron varias formas de empezar (se exponen seguidamente algunos ejemplos):

- El texto propuesto...

- Las ideas principales de los textos propuestos...

- (la idea) puede considerarse la idea principal del texto propuesto...

Pero, tras la extracción de ideas principales de los textos del examen, se debía realizar una concreción del núcleo temático. De manera que, hacía falta ofrecer un amplio abanico de posibilidades para que no todo el alumnado partiese de los mismos patrones: "Por tanto, podemos concluir que se trata del núcleo temático...", "Estas ideas permiten localizar los dos textos en el núcleo temático...", "Así deducimos que ambos textos pertenecen al núcleo temático...", entre otras comentadas en el aula. La cuestión era que sólo ofreciendo opciones al alumnado se podía conseguir que comprendiesen la importancia de iniciarse en un lenguaje y un estilo más "maduro" que el anteriormente utilizado en sus comentarios de texto.

Este trabajo se realizaba con el inicio de cada núcleo temático y tras la práctica del resumen y la síntesis. Para mejorar esta actividad de reflexión a partir de un texto dado en el momento, se les dividió en grupos de dos para que la ayuda del compañero les permitiese mejorar y ampliar su visión y su reflexión en torno al mensaje de un texto.

De acuerdo con los criterios de calificación, la redacción del resumen y del resto de información necesaria para ampliar el epígrafe a partir de los textos propuestos, sumaba un total de seis puntos. Puesto que esto ya fue trabajado como se comentó en párrafos anteriores, se insistió en la parte de integrar la información de los documentos en el grueso del núcleo temático estudiado (calificado con un punto y medio). Esta parte supuso la preparación de distintas 
fórmulas para facilitar el enlace, además de la práctica de búsquedas de información específica de los núcleos temáticos en distintos textos.

Para trabajar las fórmulas de relación, se ofrecieron diversas opciones: "como muestran los textos propuestos", "(información) que queda justificada con "(línea del texto)" (pág.)", por ejemplo. La idea era que la relación de la información quedase justificada por tres-cuatro palabras de los documentos, para así demostrar que el núcleo temático estudiado estaba comprendido porque se podían establecer relaciones con la información de los textos de la época.

Para profundizar en la relación de ideas, se recurrió a textos elegidos aleatoriamente, pero correspondientes al núcleo temático estudiado en ese momento. No se trataba de realizar el comentario de texto completo, sino que se quería trabajar únicamente las partes del núcleo temático que se podían relacionar directamente con fragmentos de los textos: se solicitó que subrayasen las líneas de texto y que marcasen con qué momento de su resumen del núcleo temático se podían establecer relaciones/inclusiones del fragmento en el mismo. Este trabajo tan compartimentado facilitó la comprensión no sólo del núcleo temático correspondiente, sino del mecanismo de evaluación y de la madurez que implica la realización de comentarios de texto.

Puesto que se destinaba medio punto a la redacción y coherencia del documento resultante, se decidió que en caso de que se realizasen faltas de ortografía, el alumnado debía copiar dicha palabra 10 veces y entregarla en un documento independiente. Así se intentó mejorar su ortografía, ya que su expresión se había pulido con las revisiones de los resúmenes y con los esquemas de respuesta para los distintos apartados del examen.

Los resultados conseguidos con esta metodología de trabajo se muestran en el siguiente epígrafe. Es cierto que no se propuso partir del uso de las nuevas tecnologías, herramientas que de acuerdo con Acosta (2010) ofrecen importantes beneficios tanto para la labor docente como para el educando, pero se consideró que debían reforzar la escritura y la expresión tradicionales porque su examen sería realizado de este modo. Colomer et al. (2004) también han investigado sobre ese uso de las nuevas tecnologías en la enseñanza de la Historia de España, aunque se puede observar que los beneficios giran más en 
torno a la preparación de los materiales de esta asignatura. En esta línea del uso tecnológico en clase de Historia, han investigado Martínez, Valls y Pineda (2009).

\section{Resultados de una experiencia práctica en un aula de $2^{\circ}$ de Bachillerato de un instituto de la Provincia de Alicante}

Durante distintos cursos académicos, siendo el primero el curso 2006/2007, se desarrollaron las clases de Historia de España de acuerdo con los parámetros comentados en el epígrafe anterior. Seguidamente, se comenta con detalle la experiencia que permitió mostrar una realidad en la que han insistido muchos investigadores como, por ejemplo, Martínez, Souto y Beltrán (2005): la necesidad de adecuarse a las exigencias de la PAU para mejorar los resultados de acceso a la universidad.

\subsection{Participantes}

La experiencia comenzó a desarrollarse en el curso académico 2006/2007 y se fue extendiendo durante los siguientes, hasta que en el curso 2008/209 se incorporó un nuevo docente, y en el curso 2009/2010 cambiaron el modelo de examen de PAU, como se ha comentado en epígrafes anteriores. Pero, para mostrar el significativo cambio en los resultados obtenidos en la asignatura de Historia de España, se han incluido los datos de los cursos 2005/2006, $2006 / 2007$ y $2007 / 2008$. Del primer curso al segundo ya se observaron ciertos cambios ascendentes, que no hacían sino aumentar durante el curso 2007/2008; aunque sólo se comentan los datos de estos períodos porque los resultados posteriores continuaron en esa línea positiva para la calificación final del alumnado y debía concretarse el momento del cambio que fue el más relevante, en cuanto a los comentarios y reflexiones del alumnado, como lo que supuso para la didáctica de la Historia en ese último curso de Bachillerato.

Durante el curso académico 2005/2006, un total de 50 alumnos de un centro de la provincia de Alicante se presentó a la PAU. De este total, sólo 27 se 
presentaron al examen de Historia de España, puesto que los restantes se presentaron al examen de Filosofía (tal como establece la legislación vigente, el alumnado puede escoger entre realizar el examen de Filosofía o el de Historia de España). El criterio del alumnado para seleccionar entre ambos exámenes se reducía a elegir aquella que pudiese aportarles un mejor resultado para su calificación final. Dado que no se veían preparados para afrontar el examen de Historia de España, puesto que no se les evaluaba a partir del examen de PAU y no lo conocían bien, optaban por Filosofía que sí era trabajada por el docente a partir del examen modelo de PAU.

En el curso 2006/2007, que fue iniciado en enero (coincidiendo con el comienzo del Bloque II "La construcción del Estado Liberal"), de un total de 50 alumnos que aprobaron en mayo, 26 se presentaron para realizar el examen de Historia de España. Debe señalarse que el trabajo tomando como eje el modelo de PAU, no se inició hasta enero debido a la incorporación al citado centro como consecuencia de una excedencia especial que duraría varios cursos académicos. Este hecho permitió desarrollar y validar el modelo didáctico propuesto, que quedaría avalado por los cambios significativos en las notas del alumnado en el mencionado examen.

Ya durante el curso académico 2007/2008, de una total de 45 alumnos aprobados para realizar la PAU en junio, 29 decidieron presentarse al examen de Historia de España.

Fue precisamente en este margen de cursos citados, en los que pudo verse la mejoría de las calificaciones del alumnado, gracias a insistir en el modelo de evaluación que el alumnado debía realizar en PAU. Éste sigue una intención muy lógica que ya señalaron autores como Martínez, Valls y Pineda (2009), que consideraban que el alumnado comprendía mejor los contenidos históricos si se trabajaba a partir de comentarios de texto. De manera que, no se podía entender la preparación de la PAU de un modo diferente, ya que así se podía no sólo mejorar la calificación del examen sino la compresión de los distintos bloques temáticos al trabajarse mediante comentarios de texto.

La siguiente tabla (Tabla 1) permite visualizar más directamente los datos de las muestras estudiadas: 
Tabla 1. Alumnado participante en la investigación

\begin{tabular}{|c|c|c|}
\hline Curso académico & Alumnado & Alumnado/Asignatura en PAU \\
\hline $2005 / 2006$ & 50 & 27 Historia de España \\
\hline $2006 / 2007$ & 50 & 26 Historia de España \\
\hline $2007 / 2008$ & 45 & 29 Historia de España \\
\hline
\end{tabular}

Fuente: elaboración propia

En el epígrafe siguiente se detallan los resultados obtenidos y la comparación entre los mismos, indicándose la subida de la nota media del centro, así como del estudiante, además de la mejora de las notas de la asignatura y la desaparición de los suspensos en la misma.

\subsection{Resultados y discusión}

En primer lugar, se van a exponer los resultados de las 27 personas examinadas de Historia de España en PAU durante el curso académico 2005/2006. La Tabla 2 muestra (en negrita) las notas más baja y más alta, así como la situación de ésta dentro de las notas de cada estudiante en el caso de que sea un suspenso. Esto permitirá comprender si fue su calificación más baja o era un alumno con calificaciones similares, lo que ayudará a comprender que el resultado bajo no fue significativo para él, dado el contexto.

Tabla 2. Resultados del Curso académico 2005/2006

\begin{tabular}{|c|c|c|}
\hline Alumnado & $\begin{array}{c}\text { Calificación Historia } \\
\text { de España }\end{array}$ & $\begin{array}{c}\text { Posición calificación Ha de España en } \\
\text { relación otras materias (sobre 7 } \\
\text { asignaturas evaluadas) }\end{array}$ \\
\hline 1 & 6,00 & $3^{\mathbf{a}}$ \\
\hline 2 & 4,50 & $6^{\mathbf{a}}$ \\
\hline 3 & 6,50 & $4^{\mathbf{a}}$ \\
\hline 4 & 7,00 & $3^{\mathbf{a}}$ \\
\hline 5 & 8,50 & $1^{\mathbf{a}}$ \\
\hline 6 & 5,50 & $6^{\mathbf{a}}$ \\
\hline 7 & 6,50 & $3^{\mathbf{a}}$ \\
\hline 8 & 4,00 & $7^{\mathbf{a}}$ \\
\hline 9 & 6,50 & $4^{\mathbf{a}}$ \\
\hline 10 & 8,00 & $4^{\mathbf{a}}$ \\
\hline 11 & 7,50 & $1^{\mathbf{a}}$ \\
\hline 12 & 7,00 & $5^{\mathbf{a}}$ \\
\hline 13 & 7,00 & $3^{\mathbf{a}}$ \\
\hline 14 & 6,00 & $5^{\mathbf{a}}$ \\
\hline
\end{tabular}




\begin{tabular}{|l|l|l|}
\hline $\mathbf{1 5}$ & $\mathbf{6 , 5 0}$ & $\mathbf{7}^{\mathbf{a}}$ \\
\hline 16 & $\mathbf{6 , 5 0}$ & $\mathbf{3}^{\mathbf{a}}$ \\
\hline 17 & $\mathbf{6 , 0 0}$ & $\mathbf{7}^{\mathbf{a}}$ \\
\hline 18 & $\mathbf{2 , 0 0}$ & $\mathbf{7}^{\mathbf{a}}$ \\
\hline 19 & $\mathbf{7 , 5 0}$ & $\mathbf{3}^{\mathbf{a}}$ \\
\hline 20 & $\mathbf{8 , 0 0}$ & $\mathbf{2}^{\mathbf{a}}$ \\
\hline 21 & $\mathbf{6 , 5 0}$ & $\mathbf{6}^{\mathbf{a}}$ \\
\hline 22 & $\mathbf{2 , 0 0}$ & $\mathbf{7}^{\mathbf{a}}$ \\
\hline 23 & $\mathbf{8 , 0 0}$ & $\mathbf{1}^{\mathbf{a}}$ \\
\hline 24 & $\mathbf{4 , 5 0}$ & $\mathbf{5}^{\mathbf{a}}$ \\
\hline 25 & $\mathbf{7 , 0 0}$ & $\mathbf{3}^{\mathbf{a}}$ \\
\hline 26 & $\mathbf{7 , 0 0}$ & $\mathbf{1}^{\mathbf{a}}$ \\
\hline 27 & $\mathbf{6 , 5 0}$ & $\mathbf{2}^{\mathbf{a}}$ \\
\hline
\end{tabular}

Fuente: elaboración propia

En segundo lugar, se expondrán los resultados del curso 2006/2007 en la siguiente tabla (Tabla 3), antes de proceder a la discusión de los resultados obtenidos:

Tabla 3. Resultados del Curso académico 2006/2007

\begin{tabular}{|c|c|c|}
\hline Alumnado & $\begin{array}{c}\text { Calificación Historia } \\
\text { de España }\end{array}$ & $\begin{array}{c}\text { Posición calificación } \mathrm{H}^{\mathrm{a}} \text { de España en } \\
\text { relación otras materias (sobre } 7 \\
\text { asignaturas evaluadas) }\end{array}$ \\
\hline 1 & 7,50 & $1^{a}$ \\
\hline 2 & 7,50 & $6^{a}$ \\
\hline 3 & 7,00 & $5^{a}$ \\
\hline 4 & 7,00 & $3^{a}$ \\
\hline 5 & 5,50 & $4^{a}$ \\
\hline 6 & 6,50 & $5^{a}$ \\
\hline 7 & 6,00 & $3^{a}$ \\
\hline 8 & 7,50 & $\mathbf{1}^{\mathrm{a}}$ \\
\hline 9 & 7,00 & $2^{a}$ \\
\hline 10 & 7,00 & $\mathbf{1}^{\mathrm{a}}$ \\
\hline 11 & 7,00 & $\mathbf{1}^{a}$ \\
\hline 12 & 6,00 & $3^{a}$ \\
\hline 13 & 8,00 & $2^{a}$ \\
\hline 14 & 6,50 & $2^{a}$ \\
\hline 15 & 8,50 & $2^{a}$ \\
\hline 16 & 6,00 & $3^{a}$ \\
\hline 17 & 7,50 & $3^{a}$ \\
\hline 18 & 8,50 & $\mathbf{1}^{\mathrm{a}}$ \\
\hline 19 & 6,50 & $\mathbf{1}^{a}$ \\
\hline 20 & 8,50 & $\mathbf{1}^{\mathrm{a}}$ \\
\hline 21 & 4,00 & $5^{a}$ \\
\hline 22 & 8,50 & $\mathbf{1}^{a}$ \\
\hline 23 & 4,00 & $5^{a}$ \\
\hline 24 & 8,50 & $1^{a}$ \\
\hline 25 & 7,00 & $4^{a}$ \\
\hline 26 & 6,50 & $3^{a}$ \\
\hline
\end{tabular}

História \& Ensino, Londrina, v. 23, n. 2, p. 59-83, jul./dez. 2017 


\section{Fuente: elaboración propia}

Ya durante el curso 2007/2008, fueron 29 los alumnos presentados al examen de Historia de España, de un total de 45 aprobados en mayo. La Tabla 4 muestra los distintos datos obtenidos:

Tabla 4. Resultados del Curso académico 2007/2008

\begin{tabular}{|c|c|c|}
\hline Alumnado & $\begin{array}{c}\text { Calificación Historia } \\
\text { de España }\end{array}$ & $\begin{array}{c}\text { Posición calificación } H^{a} \text { de España en } \\
\text { relación otras materias (sobre } 7 \\
\text { asignaturas evaluadas) }\end{array}$ \\
\hline 1 & 9,00 & $2^{a}$ \\
\hline 2 & 8,00 & $1^{a}$ \\
\hline 3 & 8,50 & $1^{a}$ \\
\hline 4 & 7,00 & $1^{a}$ \\
\hline 5 & 9,50 & $3^{a}$ \\
\hline 6 & 9,50 & $1^{a}$ \\
\hline 7 & 8,50 & $1^{a}$ \\
\hline 8 & 6,50 & $2^{a}$ \\
\hline 9 & 8,00 & $3^{a}$ \\
\hline 10 & 8,50 & $3^{a}$ \\
\hline 11 & 7,00 & $2^{a}$ \\
\hline 12 & 6,50 & $5^{a}$ \\
\hline 13 & 7,00 & $4^{a}$ \\
\hline 14 & 7,50 & $1^{a}$ \\
\hline 15 & 9,00 & $1^{a}$ \\
\hline 16 & 8,50 & $1^{a}$ \\
\hline 17 & 8,70 & $1^{a}$ \\
\hline 18 & 7,00 & $2^{a}$ \\
\hline 19 & 7,50 & $1^{a}$ \\
\hline 20 & 9,00 & $1^{a}$ \\
\hline 21 & 8,50 & $2^{a}$ \\
\hline 22 & 9,00 & $1^{a}$ \\
\hline 23 & 8,00 & $1^{a}$ \\
\hline 24 & 7,50 & $1^{a}$ \\
\hline 25 & 8,00 & $2^{a}$ \\
\hline 26 & 9,00 & $2^{a}$ \\
\hline 27 & 6,00 & $5^{a}$ \\
\hline 28 & 8,70 & $1^{a}$ \\
\hline 29 & 8,50 & $1^{a}$ \\
\hline
\end{tabular}

Fuente: elaboración propia

En la siguiente tabla (Tabla 5) se puede ver el incremento en el número de aprobados y de notables y sobresalientes de un curso a otro, tras la puesta en práctica de la metodología de trabajo centrada en el modelo de PAU: 
Tabla 5. Calificaciones de la asignatura de Historia de España dentro de los tres períodos estudiados

\begin{tabular}{|c|c|c|c|}
\hline Calificaciones & Curso 2005/2006 & Curso 2006/2007 & Curso 2007/2008 \\
\hline Menos de 5 & 5 personas & 2 personas & ---- \\
\hline $5-5,99$ & 1 persona & 1 persona & ---- \\
\hline $6-6,99$ & 10 personas & 7 personas & 3 personas \\
\hline $7-7,99$ & 7 personas & 10 personas & 7 personas \\
\hline $8-8,99$ & 4 personas & 6 personas & 12 personas \\
\hline $9-9,99$ & ---- & ---- & 7 personas \\
\hline 10 & ---- & ---- & --- \\
\hline
\end{tabular}

Fuente: elaboración propia

Se puede observar una disminución del número de suspensos y una mejora de las calificaciones, con una tendencia ascendente hacia el notable y un notable incremento de los sobresalientes. De acuerdo con esto, la Tabla 6 ilustra muy bien el cambio positivo que produjo la utilización del modelo de evaluación de PAU en el aula de $2^{\circ}$ de Bachillerato. Estos resultados muestran cómo la nota media del alumnado mejoró considerablemente gracias a la mejora de las calificaciones de Historia de España, tal como puede verse en las Tablas 2, 3 y 4. Si se hace un cálculo de la nota media de PAU obtenida por el alumnado del centro, de un curso a otro se puede observar una tendencia al alza, lo que viene a demostrar la necesidad de trabajar partiendo del modelo de evaluación de PAU.

Debe señalarse que, como puede observarse en las Tablas 2,3 y 4, en algunos casos, en los que las calificaciones de Historia de España son de bien $(6,00-6,99)$, si se observa el resto de calificaciones del alumnado, ésta se convierte en la primera o segunda mejor nota. Estos datos se han incluido en las tablas citadas, en las que se ha hecho referencia a la posición de esta nota respecto al resto de resultados de PAU:

Tabla 6. Calificaciones extremas del alumnado durante los tres períodos estudiados

\begin{tabular}{|c|c|c|c|c|c|}
\hline \multicolumn{2}{|c|}{$\begin{array}{c}\text { Curso académico } \\
2005 / 2006\end{array}$} & \multicolumn{2}{c|}{$\begin{array}{c}\text { Curso académico } \\
2006 / 2007\end{array}$} & \multicolumn{2}{c|}{$\begin{array}{c}\text { Curso académico } \\
2007 / 2008\end{array}$} \\
\hline $\begin{array}{c}\text { Calificación } \\
\text { más baja }\end{array}$ & $\begin{array}{c}\text { Calificación } \\
\text { más alta }\end{array}$ & $\begin{array}{c}\text { Calificación } \\
\text { más baja }\end{array}$ & $\begin{array}{c}\text { Calificación } \\
\text { más alta }\end{array}$ & $\begin{array}{c}\text { Calificación } \\
\text { más baja }\end{array}$ & $\begin{array}{c}\text { Calificación } \\
\text { más alta }\end{array}$ \\
\hline 2,00 & 8,50 & 4,00 & 8,50 & 6,00 & 9,50 \\
\hline
\end{tabular}

Fuente: elaboración propia 
Pese a que se mantuvo un número de suspensos (únicamente dos personas) durante el curso académico 2006/2007, es cierto que con respecto al curso académico 2005/2006, la nota de suspenso más baja fue un 4,00, frente a una persona con 2,00, otra con 4,00 y dos personas con 4,50. $Y$ ya en el curso 2007/2008 terminaron por desaparecer los suspensos en la asignatura, tendencia que continuaría en cursos posteriores. Además, en este curso 2007/2008, la nota más baja pasaría a ser un 6,00 (una sola persona obtuvo esta calificación más baja).

Una situación similar se daría con las calificaciones más altas. Frente a un 8,50 conseguido por una única persona, serían cinco las que obtendrían esta nota en el curso académico 2006/2007; y ya en 2007/2008, dos personas conseguirían 9,50 en este examen de Historia de España, subiendo la nota media de la asignatura.

Junto a todo esto, debe señalarse que la calificación de Historia de España, dentro del grueso de calificaciones del resto de asignaturas examinadas en la citada PAU a lo largo de los distintos cursos académicos, ha mejorado. Esto es que, si en el curso 2005/2006 sólo cuatro personas contaban su nota de Historia de España como la primera mejor nota, en el curso siguiente (2006/2007) ésta pasaría a ser la mejor calificación para nueve personas, frente a las 16 personas que la contarían como su mejor nota en el curso académico 2007/2008. En este sentido, las diferencias son significativas, ya que al mejorarse los resultados e ir aumentando la calificación numérica de la misma, el hecho de que muchos alumnos la tengan como su mejor nota indica que fue una de las mejor preparadas, ya que obtuvieron mejores calificaciones.

Señalemos que el alumnado consideraba que si durante el curso siguiente (2008/2009) estudiaba con el resumen de un amigo que hubiese obtenido buena nota en PAU durante el curso 2007/2008, también obtendrían buena nota. Sin embargo, se trataba del proceso para llegar a ese resumen, el trabajo separado y profundo de cada parte del examen, lo que hizo que las notas mejorasen, tal como se puede comprobar por los resultados mostrados en páginas anteriores. En este sentido, el docente que se encargó del alumnado de Historia de España del centro en cuestión recogió el testigo en cuanto al trabajo según el modelo de examen, lo que permitió replicar hasta cierto punto los resultados; ahora bien, el 
cambio de modelo de examen durante el curso 2009/2010 y la falta de planteamiento de una nueva metodología adaptada al mismo, supuso una bajada de notas del alumnado de dicho centro que tenía que realizar este examen de forma obligatoria (durante los cursos investigados se escogía entre Historia de la Filosofía e Historia de España).

\section{Conclusiones}

La didáctica de la Historia puede haber permitido muchas investigaciones, la aparición de mucha bibliografía especializada, pero todo esto puede quedar más o menos en el olvido cuando el alumnado se enfrenta a un modelo de evaluación muy específico con una clara intencionalidad de mostrar que la capacidad de síntesis y el raciocinio por parte del alumnado, son una realidad. García, Jiménez y Moreno (2011) han investigado sobre los cambios en el modelo de examen y sus criterios de evaluación, que considera llevan al alumnado a un proceso de reflexión sobre la conciencia histórica: no obstante, el modelo de cursos anteriores (hasta el curso 2008/2009) que se ha analizado en esta investigación, por su estructura y exigencias, se puede decir sin duda que promueve mayor reflexión del alumnado que el surgido a partir del curso 2009/2010, tal como se ha comentado anteriormente.

La PAU pretende que el alumnado se examine no sólo de aquellos conocimientos que a nivel gubernamental se han considerado imprescindibles para el alumnado, sino que éste consiga desarrollar su capacidad de síntesis, de discernimiento, de razonamiento..., a partir de documentos relacionados con los distintos temas estudiados. Precisamente por esto, el examen de Historia de España no parte del modelo tradicional de desarrollar uno o dos temas, sino que se pretende que el alumnado parta de dos documentos significativos de un período estudiado, y que a partir de ellos se elabore un "texto" que permita no sólo mostrar los conocimientos estudiados sino también establecer relaciones y vínculos entre los dos textos y el tema relacionado. Pero esto no sucede sólo en la Comunidad Valenciana, ya que Martínez, Conejo y López (2011) han investigado sobre este examen en Galicia, comprendido como promotor del 
fomento de competencias analíticas y personales, en la misma línea de la Comunidad Valenciana. Suárez (2010) también defiende que, para lograr el aprendizaje del alumnado, sea necesario establecer relaciones entre la información nueva y la previa, como se promovía en la PAU de la citada comunidad autónoma antes del curso 2009/2010. A esta conclusión también ha llegado Fuster (2016), que considera que la actual PAU no permite una interpretación de la realidad social sino sólo una potenciación de la memorización de contenidos.

En este sentido, por eso es imprescindible que el alumnado trabaje con las pautas indicadas, ya que sólo así podrá conseguirse no sólo el demostrado incremento de la nota del alumnado en este examen concreto de Historia de España, sino que al mismo tiempo éste pueda conseguir razonar, discernir y comprender su pasado, su presente y así pueda decidir su futuro.

\section{Referencias}

ACOSTA, Luis Miguel. La enseñanza-aprendizaje de la historia en bachillerato y las TIC: la introducción de la estrategia Webquest. Clío, Barcelona, n. 36, p. 110, 2010.

ALARCÓN, Ma Dolores. Las PAU de Historia de España en el año 2009: un estudio descriptivo por Comunidades Autónomas. Memoria Docente e Investigadora no publicada. Murcia: Universidad de Murcia, 2010.

COLOMER, Francisca et al. La ausencia de las TIC's en la enseñanza de la Historia de España en Bachillerato LOGSE y COU. Notas de una investigación en curso realizada en la Comunidad Autónoma de Murcia. In: VERA, Ma Isabel; PÉREZ, David (Coord.). Formación de la ciudadanía: las TIC's y los nuevos problemas. Alicante: Asociación Universitaria de Profesores de Didáctica de las Ciencias Sociales, 2004. p. 1-12.

ESPANHA. Jefatura Del Estado. Ley Orgánica 2/2006, de 3 de mayo, de Educación. BOE (04/05/2006), no 106, referencia 7899, p. 17158-17207, 2006.

ESPANHA. Jefatura Del Estado. Ley Orgánica 8/2013, de 9 de diciembre, para la mejora de la calidad educativa. BOE (10/12/2013), n 295, referencia 12886, p. 97858-97921, 2013.

ESPANHA. Ministerio De Educación Y Ciencia. Real Decreto 1467/2007, de 2 de noviembre. Se establece la estructura del bachillerato y se fijan sus enseñanzas mínimas. $B O E(06 / 11 / 2007)$, no 266, referencia 19184, p. 45381-45477, 2007. 
ESPANHA. Ministerio De La Presidencia. Real Decreto 1892/2008, de 14 de noviembre. Se regulan las condiciones para el acceso a las enseñanzas universitarias oficiales de grado y los procedimientos de admisión a las universidades públicas españolas. $B O E(24 / 11 / 2008)$, no 283, referencia 18947, pp. 46932-46946.

FUSTER, Carlos. Pensar históricamente. La evaluación en la PAU de Historia de España. Valencia: Universidad de Valencia, 2016.

GAETE, Verónica. Desarrollo psicosocial del adolescente. Revista Chilena de Pediatría, Santiago de Chile, v. 6, n. 86, p. 436-443, 2015.

GARCÍA, Antonio Luis; LARA, Juan Jesús. La enseñanza de la Geografía en Bachillerato: datos y consideraciones sobre sus resultados en Selectividad. Cuadernos Geográficos, Merida, v. 1, n. 44, p. 193-232, 2009.

GARCÍA, Carmen Rosa; JIMÉNEZ, Ma Dolores; MORENO, Concepción. Las pruebas de acceso a la universidad (PAU) de Historia de España. Una indagación sobre la formación del pensamiento histórico de los estudiantes. In: MIRALLES, Pedro; MOLINA, Sebastián; SANTISTEBAN, Antoni (Ed.). La evaluación en el proceso de enseñanza y aprendizaje de las ciencias sociales. Murcia: Fundación Séneca-Agencia de Ciencia y Tecnología de la Región de Murcia y Ministerio de Ciencia e Innovación, 2011. p. 203-212.

GÓMEZ, Cosme Jesús; MIRALLES, Pedro. La enseñanza de la historia desde un enfoque social. Clío, Barcelona, n. 39, p. 1-16, 2013.

HAZEN, Eric; SCHOLZMAN, Steven; BERESIN, Eugene. Adolescent psychological development: A review. Pediatrics in Review, Evanston, n. 29, p. 161-168, 2008.

INAREJOS, Juan Antonio. Nacionalismos e identidades en la enseñanza de la historia de España: avances y resistencias. Clío, Barcelona, n. 39, p. 1-12, 2013.

LLÁCER, Vicent. La enseñanza de la historia desde la perspectiva del profesorado de bachillerato. Didáctica de las Ciencias Experimentales y Sociales, Valencia, $\mathrm{n}$. 21, p. 53-89, 2007.

MARTÍNEZ, Nicolás; SOUTO, Xosé Manuel; BELTRÁN, José. Los problemas docentes en historia de bachillerato: la gran diversidad bajo la aparente uniformidad. Didáctica de las Ciencias Experimentales y Sociales, Valencia, n. 19, p. 33-55, 2005.

MARTÍNEZ, Nicolás; VALLS, Rafael; PINEDA, Francisco. El uso del libro de texto de Historia de España en Bachillerato: diez años de estudio, 1993-2003, y dos reformas (LGE-LOGSE). Didáctica de las Ciencias Experimentales y Sociales, Valencia, n. 23, p. 3-35, 2009.

MARTÍNEZ, Rosendo; CONEJO, Francisco; LÓPEZ, Ramón. La evaluación en las PAU de Historia: un estudio de la transición a la democracia en España. In: MIRALLES, Pedro; MOLINA, Sebastián; SANTISTEBAN, Antoni. (Ed.). La evaluación en el proceso de enseñanza y aprendizaje de las ciencias sociales. 
Murcia: Fundación Séneca-Agencia de Ciencia y Tecnología de la Región de Murcia y Ministerio de Ciencia e Innovación, 2011. p. 259-268.

ORGANIZACIÓN MUNDIAL DE LA SALUD. Programming for adolescent health and development: report of a WHO/UNFPA/UNICEF Study Group on Programming for Adolescent Health. Geneva, 1999.

ORTIZ, Eva; MILLARES, Pedro. El uso de los apuntes en la enseñanza de la historia en la Comunidad Autónoma de la Región de Murcia. Clío, Barcelona, n. 16, p. 114-126, 2012.

PÉREZ, Amparo. La prueba de Historia de España del bachillerato LOGSE: un caso particular especialmente significativo. Tarbiya, Madrid, v. 2, n. 43, p. 177193, 2014.

PÉREZ, Antonio; GUITIÁN, Carlos. La Historia de España en el Bachillerato. ¿Contribuye su estudio a la comprensión de los grandes problemas y preocupaciones de la sociedad? In: GÓMEZ, Antonio Ernesto; NúÑEZ, Ma Pilar (Ed.). Formar para Investigar, Investigar para Formar en Didáctica de las Ciencias Sociales. Málaga: Asociación Universitaria del Profesorado de Didáctica de las Ciencias Sociales, 2006. p. 177-186.

SUÁREZ, Miguel Ángel. Enseñanza de la Historia: viejos problemas y necesidad de un cambio. Reflexión de un alumno del Máster de Profesorado de Secundaria. Clío, Barcelona, n. 36, p. 1-10, 2010.

VALENCIANA. Conselleria De Educación. Decreto 102/2008, de 11 de julio, del Consell, por el que se establece el currículo del bachillerato en la Comunitat Valenciana. DOGV (15/07/2008), no 5806, referencia 8761, p. 71303-71547, 2008. 